\title{
INCIDÊNCIA DE AFLATOXINAS E FUMONISINAS EM PRODUTOS DE MILHO CONSUMIDOS NO ESTADO DE MINAS GERAIS
}

\author{
PRISCÍULA FERREIRA ${ }^{1}$, VALÉRIA APARECIDA VIEIRA QUEIROZ², \\ RENATA REGINA PEREIRA DA CONCEIÇÃO ${ }^{3}$ RAFAEL DE ARAÚJO MIGUEL²
}

${ }^{1}$ Unifenas, Belo Horizonte, MG, Brasil, pris7l@yahoo.com.br

${ }^{2}$ Embrapa Milho e Sorgo, Sete Lagoas, MG, Brasil,valeria.vieira@embrapa.br,rafael.miguel@embrapa.br

${ }^{3}$ Unifemm, Sete Lagoas, MG, Brasil, renataponts@hotmail.com

Revista Brasileira de Milho e Sorgo, v.12, n.1, p. 54-60, 2013

\begin{abstract}
RESUMO - As aflatoxinas e as fumonisinas são micotoxinas usualmente encontradas em milho e, quando ingeridas, são capazes de provocar malefícios à saúde humana e animal. Assim, objetivou-se avaliar a incidência de aflatoxinas e de fumonisinas em seis marcas de canjiquinha, cinco de fubá e cinco de farinha de milho comercializadas em quatro supermercados do município de Sete Lagoas, MG. As análises foram realizadas por fluorimetria, utilizando-se colunas de imunoafinidade AflaTest e FumoniTest para as análises de aflatoxinas e fumonisinas, respectivamente. Dentre os produtos analisados, o fubá apresentou contaminação com ambas micotoxinas em todas as marcas, com valores que variaram entre $0,04 \mu \mathrm{g} \mathrm{kg}^{-1}$ e $1,6 \mu \mathrm{g} \mathrm{kg}^{-1}$ e entre 55 e $985 \mu \mathrm{g} \mathrm{kg}^{-1}$ para aflatoxinas e fumonisinas, respectivamente. A farinha de milho apresentou pequena contaminação com aflatoxinas $\left(0,36-0,49\right.$ e $\left.0,181 \mu^{g^{2} g^{-1}}\right)$ em três marcas e de fumonisinas $\left(20 \mu \mathrm{g} \mathrm{kg}^{-1}\right.$ e $\left.37 \mu \mathrm{g} \mathrm{kg}^{-1}\right)$ em duas marcas. Foi detectada presença de aflatoxina em três marcas de canjiquinha, variando entre 0,008 e $0,21 \mu \mathrm{g} \mathrm{kg}^{-1}$, e de fumonisina em apenas uma com $60 \mu \mathrm{g} \mathrm{kg}^{-1}$. Os produtos analisados apresentaram concentrações abaixo dos limites máximos tolerados no Brasil para ambas as micotoxinas, não oferecendo riscos para a saúde dos consumidores.
\end{abstract}

Palavras-chave: Aspergillus sp.; Fusarium sp.; micotoxinas.

\section{INCIDENCE OF AFLATOXINS AND FUMONISINS IN CORN-BASED PRODUCTS CONSUMED IN THE STATE OF MINAS GERAIS}

\begin{abstract}
Aflatoxins and fumonisins are mycotoxins commonly found in corn, and when ingested can cause harm to human and animal health. Thus, the objective of this study was to evaluate the incidence of aflatoxins and fumonisins in six brands of corn grits, five of cornmeal and five of corn flour, marketed by four supermarkets in Sete Lagoas, MG. Analyses were performed by fluorimetry method using the AflaTest and FumoniTest immunoaffinity columns for aflatoxins and fumonisins analyses, respectively. Corn flour of all brands were contamined by both mycotoxins, with values ranging from $0.04 \mathrm{mg} \mathrm{kg}^{-1}$ and $1.6 \mathrm{mg} \mathrm{kg}^{-1}$ and between 55 and $985 \mathrm{mg} \mathrm{kg}^{-1}$ for aflatoxins and fumonisins, respectively. Corn flour presented little aflatoxin contamination ( 0.36 to 0.49 and $\left.0.181 \mathrm{mg} \mathrm{kg}^{-1}\right)$ in three brands, and fumonisins $\left(20 \mathrm{mg} \mathrm{kg}^{-1}\right.$ and $\left.37 \mathrm{mg} \mathrm{kg}^{-1}\right)$ in two brands. Aflatoxin was detected in three of the evaluated corn grits, varying between 0.008 and $0.21 \mathrm{mg} \mathrm{kg}^{-1}$ and fumonisin was present in only one with $60 \mathrm{mg} \mathrm{kg}^{-1}$. The products analyzed presented concentrations below the maximum tolerated in Brazil for both mycotoxins, therefore not harmful to the consumer's health.
\end{abstract}

Key words: Aspergillus sp.; Fusarium sp.; mycotoxins. 
O Brasil é o terceiro produtor mundial de milho, com uma expectativa de produção de mais de 69 milhões de toneladas na safra 2011/2012 (Conab, 2012). Em 2010, Minas Gerais ficou em terceira posição no ranking nacional, com uma produção de cerca de seis milhões de toneladas do cereal (IBGE, 2012).

Além da produção, o estado também ocupa posição de destaque no consumo de milho e de seus derivados desde a época da colonização. Segundo Cavalieri (2011), a importação de alimentos de outros estados já povoados, como o Rio de Janeiro e a Bahia, era muito difícil. Assim, o milho, alimento mais consumido pelos indígenas, passou a ser utilizado também no preparo de pratos, tanto de escravos, como de portugueses, tornando-se um alimento universal na cozinha mineira, o que predomina até hoje. O cereal, considerado como "ouro em pencas" pelos mineiros, pode ser utilizado no preparo de diversos pratos, entre outros, a broa, o angu, a canjiquinha, o mingau de milho verde, a canjica doce, a pamonha e o corá (Cavalieri, 2011).

Entretanto, a elevada suscetibilidade à ocorrência de fungos toxigênicos, aliada à predominância de clima tropical no país, favorecem aumento da síntese de micotoxinas no milho e em seus derivados. As micotoxinas são metabólitos secundários produzidos por fungos, que podem causar efeitos agudos ou crônicos à saúde humana e de animais (Santurio, 2000). Por isto, sua presença em alimentos constitui problema de saúde pública em nível mundial (Rocha et al., 2008).

As de maior incidência em milho são sintetizadas, principalmente, por fungos dos gêneros Fusarium (fumonisinas e zearalenona), Aspergillus (aflatoxinas e ocratoxina) e Penicillium (ocratoxina) (Kawashima \& Valente Soares, 2006). As aflatoxinas B1, B2, G1 e G2 são originadas, principalmente, pelas espécies Aspergillus flavus e Aspergillus parasiticus e, devido à alta toxidez e à ampla ocorrência, são consideradas as micotoxinas causadoras dos danos mais severos à saúde de seres humanos e animais, possuindo propriedades carcinogênicas, mutagênicas, teratogênicas e imunossupressoras (Lazo, 2008). Já as fumonisinas são prevalentemente produzidas por Fusarium verticillioides, Fusarium proliferatum e Fusarium nygamai, além de Alternaria alternata $f$. sp. lycopersici (Freire et al., 2007). Podem causar leucoencefalomalácia em equinos, edema pulmonar em suínos e têm sido epidemiologicamente associadas ao câncer de esôfago em humanos (Ottoni, 2008), sendo classificadas como possíveis carcinogênicas para o homem (International Agency for Research on Cancer, 2002).

A contaminação dos alimentos pode ocorrer no campo, durante e após a colheita, no transporte, no processamento e no armazenamento do produto. Existem duas formas de inserção das micotoxinas nas cadeias alimentares: por contaminação direta, quando o fungo toxigênico contamina o produto e, em sequência, sintetiza a micotoxina e ambos permanecem no produto; e por contaminação indireta, em que a micotoxina estará presente no produto final resultante de contaminação fúngica prévia, porém o fungo foi eliminado no processamento (Freire et al., 2007).

A incidência de micotoxinas em milho e em produtos derivados tem sido relatada em estudos realizados em vários estados brasileiros (Machinski \& Valente Soares, 2000; Van Der Westhuizen, 2003; Bittencourt et al., 2005; Amaral et al., 2006; Kawashima \& Valente Soares, 2006; Cruz, 2010). Porém, poucos foram os trabalhos realizados com os produtos mais consumidos e comercializados no estado de Minas Gerais. Assim, devido aos potentes prejuízos que essas substâncias podem causar para o agronegócio e para a saúde humana e ao elevado consumo de produtos à base de milho em Minas Gerais, o objetivo deste trabalho foi avaliar 
a incidência de aflatoxinas e fumonisinas totais em canjiquinha, fubá e farinha de milho comercializados no município de Sete Lagoas, estado de Minas Gerais.

\section{Material e Métodos}

Para a realização do trabalho, foram selecionados, aleatoriamente, quatro supermercados do município de Sete Lagoas, Região Central de Minas Gerais, onde ocorreu, entre fevereiro e março de 2010, a coleta de cinco amostras de fubá, cinco de farinha de milho e seis de canjiquinha de marcas variadas, totalizando 16 amostras. Após a coleta, as amostras foram levadas ao Laboratório de Segurança Alimentar da Embrapa Milho e Sorgo, localizada em Sete Lagoas, MG, e armazenadas a $-18^{\circ} \mathrm{C}$ até o momento das análises. As análises de aflatoxinas e fumonisinas foram realizadas em fluorímetro VICAN, Séries-4, com purificação prévia das amostras em colunas de imunoafinidade AflaTest e FumoniTest, respectivamente, de acordo com procedimentos preconizados nos manuais de análise de aflatoxinas e fumonisinas da VICAN. As análises foram realizadas em triplicata.

As amostras foram homogeneizadas em quarteador em Y e uma fração de $20 \mathrm{~g}$ foi pesada em balança analítica (OHAUS, modelo AR3130) e transferida para erlenmeyer de $250 \mathrm{ml}$, onde foram adicionados $2 \mathrm{~g}$ de cloreto de sódio $(\mathrm{NaCl})$ e 40 $\mathrm{ml}$ de solução metanol: água $(80: 20 \mathrm{v} / \mathrm{v})$. Os frascos foram levados para agitação em mesa agitadora (Nova Ética, modelo 109) por 2 minutos a $200 \mathrm{rpm}$ e, em seguida, seu conteúdo foi filtrado em papel qualitativo. Após essa etapa, os procedimentos para as análises de aflatoxinas e de fumonisinas se diferenciaram, conforme descrito a seguir.

\section{Análise de aflatoxinas totais}

Pipetaram-se $5 \mathrm{ml}$ do filtrado para um béquer, adicionaram-se $20 \mathrm{ml}$ de água deionizada e, após homogeneização e filtração em filtro de microfibra de

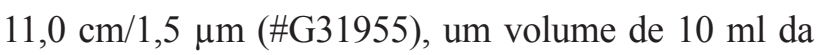
solução foi aplicado em coluna de imunoafinidade Aflatest ${ }^{\circledR}(\mathrm{VICAN})$. Em seguida, o conteúdo da coluna foi lavado com $10 \mathrm{ml}$ de água deionizada por duas vezes. As aflatoxinas foram eluídas com 1,0 ml de metanol, grau HPLC. Ao eluato, adicionou-se 1,0 $\mathrm{ml}$ de solução reveladora para aflatoxinas (VICAN) e, após 4 min, a concentração de aflatoxinas totais foi obtida em fluorímetro VICAN Serie-4, previamente calibrado.

\section{Análise de fumonisinas totais}

Pipetaram-se $5 \mathrm{ml}$ do filtrado para um béquer, adicionaram-se $20 \mathrm{ml}$ de tampão PBS/0,1\% Tween 20 e, após homogeneização e filtração em filtro de microfibra de 9,0 cm/1,0 $\mu \mathrm{m}$ (\#G2005), um volume de 10 $\mathrm{ml}$ da solução foi aplicado em coluna de imunoafinidade Fumonitest ${ }^{\circledR}$ (VICAN). Em seguida, o conteúdo da coluna foi lavado com $10 \mathrm{ml}$ de tampão $\mathrm{PBS} / 0,1 \%$ Tween 20 por duas vezes. As fumonisinas foram eluídas com 1,0 ml de metanol, grau HPLC. Ao eluato, adicionou-se 1,0 $\mathrm{ml}$ de solução reveladora para fumonisina (VICAN) e, após 4 min, a concentração de fumonisinas totais foi obtida em fluorímetro VICAN Serie-4, previamente calibrado.

$\mathrm{Na}$ etapa de extração das amostras de farinha de milho, utilizou-se volume de $80 \mathrm{ml}$ de metanol $80 \%$ e $20 \mathrm{~g}$ de amostra e, ao final da análise, dobraram-se os valores obtidos.

Os dados foram avaliados por Anova e as médias comparadas pelo teste de Tukey a 5\% de probabilidade. 


\section{Resultados e Discussão}

Os resultados dos teores de aflatoxinas totais e fumonisinas totais nas amostras analisadas encontram-se nas Figuras 1 e 2, respectivamente. Não foi detectada contaminação com aflatoxinas nos produtos fubá de milho da marca 5, farinha de milho das marcas 3 e 5 e canjiquinha das marcas 1, 2, 3, 4 e 5. Da mesma forma, não se observou contaminação com fumonisinas nas farinhas de milho das marcas 1,4 e 5 e nas canjiquinhas de milho das marcas de 1 a 5 .

Verificou-se maior índice de contaminação com aflatoxinas no produto fubá de milho, cujas cinco marcas analisadas apresentaram teores entre $0,04 \mu \mathrm{g}$ $\mathrm{kg}^{-1}$ (marca 2) e 1,6 $\mu \mathrm{g} \mathrm{kg}^{-1}$ (marca 1). Apenas três marcas de farinha de milho (1, 2 e 4) e de canjiquinha (4, 5 e 6) estavam contaminadas com aflatoxinas, com valores variando entre 0,18 e 0,49 e entre 0,008 e $1,1 \mu \mathrm{g}$ $\mathrm{kg}^{-1}$, respectivamente.

Semelhante ao que foi observado para aflatoxinas, os teores detectados de fumonisina foram maiores no produto fubá de milho, cujas cinco amostras se encontravam contaminadas com valores entre 55 e 985 $\mu \mathrm{g} \mathrm{kg}{ }^{-1}$. Apenas as marcas 2 e 3 de farinha de milho apresentaram contaminação com essa micotoxina entre $20 \mu \mathrm{g} \mathrm{kg}^{-1}$ e de $37 \mu \mathrm{g} \mathrm{kg}^{-1}$, respectivamente. No produto canjiquinha de milho, foi detectada presença de fumonisinas apenas na marca 6 , com teor de $60 \mu \mathrm{g} \mathrm{kg}^{-1}$.

Os resultados obtidos mostraram contaminação por aflatoxinas em 10 de 16 amostras analisadas, totalizando $62,5 \%$, e por fumonisinas em oito amostras, ou seja, em 50\% delas. Apesar da maioria dos produtos apresentarem contaminação, os níveis se encontravam abaixo dos limites máximos tolerados (LMT) no Brasil (Brasil, 2011), tanto para aflatoxinas totais $\left(20 \mu \mathrm{g} \mathrm{kg}^{-1}\right)$, quanto para fumonisinas totais (2000 a $2500 \mu \mathrm{g} \mathrm{kg}^{-1}$ ).
Corroborando esses resultados, Machinsky \& Valente Soares (2000) avaliaram 81 alimentos à base de milho comercializados na cidade de Campinas, SP, e detectaram contaminação com as fumonisinas B1 em 40 e B2 em 44 amostras. Por outro lado, Scaff \& Scussel (2004) analisaram a incidência de fumonisinas B1 e B2 em farinha de milho, em milho de pipoca e em canjica, comercializados em Santa Catarina, e verificaram que $92,68 \%$ das amostras encontravam-se com teores detectáveis de fumonisina, sendo que a farinha de milho foi o produto que obteve o maior índice de contaminação, diferente dos resultados obtidos neste trabalho, o qual apresenta o fubá com maior incidência dessa micotoxina.

No que tange à presença de aflatoxinas, Marques (2007) também encontrou resultados semelhantes ao presente trabalho em amostras comerciais de quirera, as quais encontravam-se com teores abaixo de $20 \mu \mathrm{g} \mathrm{kg}^{-1}$. Da mesma forma, Amaral et al. (2006) observaram baixa ocorrência de aflatoxinas nos produtos à base de milho analisados em Maringá e em Marialva, ambas cidades paranaenses. Entretanto, os autores concluíram que a Ingestão Diária Provável Média (IDPM) de aflatoxina B1 foi acima da Ingestão Diária Tolerável (IDT), indicando risco para a população da Região Sul do Brasil devido ao maior consumo de produtos à base de milho naquela região.

Segundo Moraes (2009), os produtos farinha, canjiquinha e fubá de milho são obtidos a partir do processo de moagem do milho a seco, no qual o endosperma, o pericarpo e o gérmen do milho são separados por meio mecanizado e o pericarpo se transforma em um resíduo farináceo, utilizado principalmente para alimentação animal. O pericarpo é a parte mais externa do grão e, por possuir estrutura mais rígida, constitui barreira física contra a entrada 


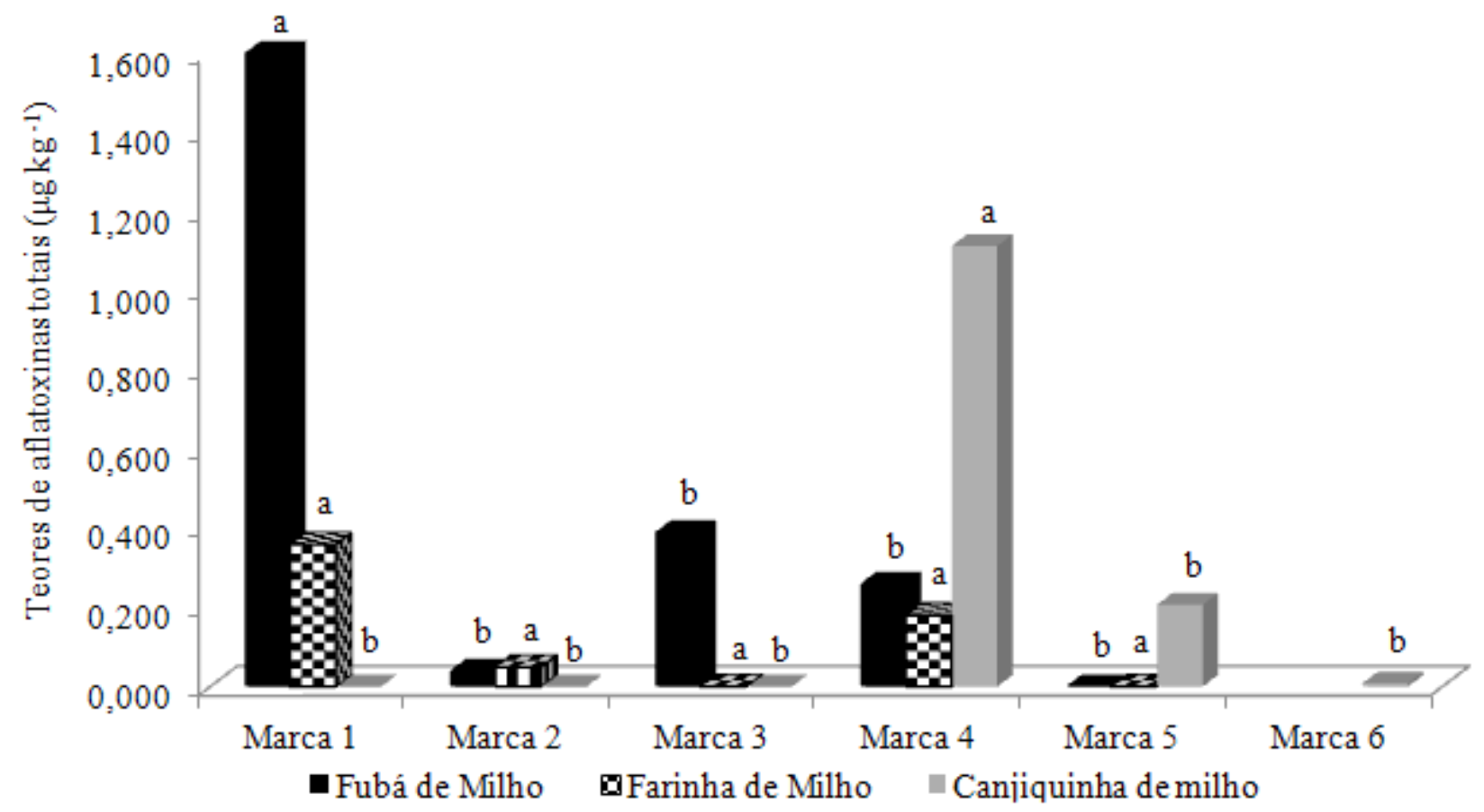

FIGURA 1. Teores de aflatoxinas totais $\left(\mu \mathrm{g} \mathrm{kg}^{-1}\right)$ em fubá de milho, farinha de milho e canjiquinha de milho de seis diferentes marcas comerciais.

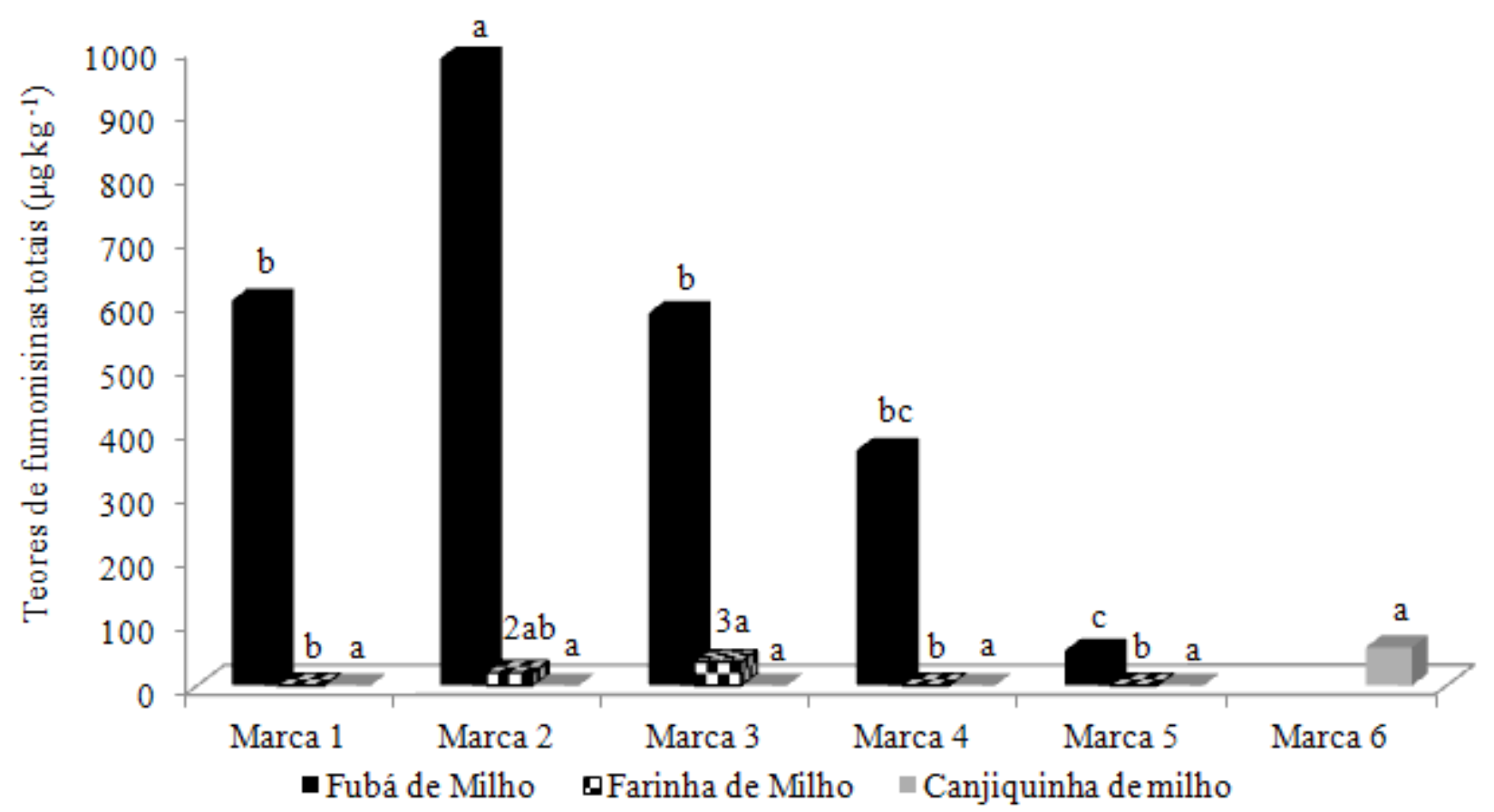

FIGURA 2. Teores de fumonisinas totais $\left(\mu \mathrm{g} \mathrm{kg}^{-1}\right)$ em fubá de milho, farinha de milho e canjiquinha de milho de seis diferentes marcas comerciais. 
de fungos no endosperma, podendo contribuir para redução nos teores de micotoxinas nos produtos processados em relação aos grãos inteiros. Essa pode ser uma possível explicação para os baixos teores de micotoxinas detectados nos produtos analisados no presente trabalho e em outros (Cruz, 2010; Marques, 2007). Corroborando esse argumento, Scudamore \& Patel (2000) observaram que uma limpeza inicial das amostras de milho realizada após moagem dos grãos permitiu redução de $40 \%$ na concentração de aflatoxinas e de $32 \%$ de fumonisinas em relação aos teores iniciais detectados nessas mesmas amostras.

\section{Conclusões}

Embora 10 das 16 amostras analisadas apresentassem contaminação com aflatoxinas $(62,5 \%)$ e oito amostras com fumonisinas (50\%), os teores detectados nos produtos fubá, farinha de milho e canjiquinha de milho encontravam-se abaixo dos limites máximos tolerados para ambas as micotoxinas no Brasil. Assim, esses produtos, referentes aos lotes analisados, não oferecem risco à saúde humana.

Apesar dos níveis dessas toxinas se apresentarem baixos neste trabalho, deve haver monitoramento constante desses produtos à base de milho, visto ser a contaminação por micotoxinas dependente de um conjunto de fatores.

\section{Agradecimentos}

Ao Conselho Nacional de Desenvolvimento Científico e Tecnológico (CNPq), pela bolsa de iniciação científica. À Embrapa Milho e Sorgo, pela oportunidade de estágio. À Solcampo, pela doação das colunas de imunoafinidade FumoniTest ${ }^{\circledR}$ e AflaTest ${ }^{\circledR}$ utilizadas nas análises de fumonisinas.

\section{Referências}

AMARAL, K. A. S.; NASCIMENTO, G. B.; SEKIYAMA, L.; JANEIRO, V.; MACHINSKI JR, M. Aflatoxinas em produtos a base de milho comercializados no Brasil e riscos para a saúde humana. Ciência e Tecnologia de Alimentos, Campinas, v. 26, p. 336342, 2006.

BITTENCOURT, A. B. F.; OLIVEIRA, C. A. F.; DILKIN, P.; CORRÊA, B. Mycotoxin occurrence in corn meal and flour traded in Sao Paulo, Brazil. Food Control, Guildford, v. 16, p. 117-120, 2005.

BRASIL. Ministério da Saúde. Agencia Nacional de Vigilância Sanitária. Regulamento técnico sobre limites máximos tolerados (LMT) para micotoxinas em alimentos. Resolução RDC $\mathrm{n}^{\circ} 7$, de 18 de fevereiro de 2011. Brasília, DF: ANVISA, 2011.

CAVALIERI, R. B. Culinária Mineira: as raízes históricas da sua diversidade. 15 maio 2010. Disponível em: $<$ http://mgculinariamineira.blogspot.com/2010/05/ culinaria-mineira-as-raizes-historicas.html $>$. Acesso em: 3 ago. 2011.

CONAB. Acompanhamento da safra brasileira: grãos: décimo levantamento, julho/2012. Brasília, DF, 2012. p. 22. Disponível em: < http://www.conab.gov.br/ OlalaCMS/uploads/arquivos/12_07_05_08_41_20 boletim_graos_-_10 julho_2012.pdf >. Acesso em: 3 ago. 2012.

CRUZ, J. V. Ocorrência de aflatoxinas e fumonisinas em produtos à base de milho e milho utilizado como ingrediente de ração para animais de companhia comercializados na região de Pirassununga, Estado de São Paulo. 2010. 73 f. Tese (Doutorado em Zootecnia) - Faculdade de Zootecnia e Engenharia de Alimentos, Universidade de São Paulo, Pirassununga. 
FREIRE, F. C. O.; VIEIRA, I. G. P.; GUEDES, M. I. F.; MENDES, F. N. P. Micotoxinas: importância na alimentação e na saúde humana e animal. Fortaleza: Embrapa Agroindústria Tropical, 2007. 48 p. (Embrapa Agroindústria Tropical. Documentos, 110).

IBGE. Banco de Dados Agregados. Sistema IBGE de Recuperação Automática - SIDRA: tabela 839 Área plantada, área colhida, quantidade produzida e rendimento médio de milho, $1^{\mathrm{a}}$ e $2^{\mathrm{a}}$ safras: ano 2010. Disponível em: <http://www.sidra.ibge.gov.br/bda/ tabela/listabl.asp? $\mathrm{z}=\mathrm{t} \& \mathrm{c}=839>$. Acesso em: 7 ago. 2012.

INTERNATIONAL AGENCY FOR RESEARCH ON CANCER. Some Traditional Herbal Medicines, Some Mycotoxins, Naphthalene and Styrene. Lyon, 2002. (IARC monographs on the evolution of carcinogenic risks to humans, 82).

KAWASHIMA, L. M.; VALENTE SOARES, L. M. Incidência de fumonisina B1, aflatoxinas B1, B2, G1 e G2, ocratoxina A e zearalenona em produtos de milho. Ciência e Tecnologia de Alimentos, Campinas, v. 26, p. 516-521, 2006.

LAZO, R. F.; SIERRA, G. Investigacion del efecto de las micotoxinas en el ser humano. Revista Iberoamericana de Micologia, Madri, v. 25, p. 7-11, 2008

MACHINSKI JR, M.; VALENTE SOARES, L. M. Fumonisins B1 and B2 in Brazilian corn-based food products. Food Additives and Contaminants, London, v. 17, p. 875-879, 2000.

MARQUES, P. J. N. Avaliação de aflatoxina e zearalenona em quirera (canjiquinha de milho) na região dos Campos Gerais. 2007. 96 f. Dissertação
(Mestrado em Ciência e Tecnologia de Alimentos)

- Universidade Estadual de Ponta Grossa, Ponta Grossa.

MORAES, V. B. Efeito do resíduo da moagem à seco de milho, micropulverizado no metabolismo lipídico, na glicemia e na composição corporal em ratos alimentados com dieta da cafeteria. 2009. $64 \mathrm{f}$. Dissertação (Mestrado em Ciência da Nutrição) Universidade Federal de Viçosa, Viçosa.

OTTONI, J. R. Análise da incidência de Fusarium spp. toxigênico e de níveis de fumonisina em grãos ardidos de milho híbrido. 2008. 55 f. Dissertação (Mestrado em Microbiologia Agrícola) - Escola Superior de Agricultura Luiz de Queiroz, Universidade de São Paulo, Piracicaba.

ROCHA, M. D.; MAIA, P. P.; RODRIGUES, M. A. C.; MARTINS, I. Incidência de aflatoxinas em amostras de amendoim e paçoca comercializadas na cidade de Alfenas-MG, Brasil. Revista Brasileira de Toxicologia, São Paulo, v. 21, p. 15-19, 2008.

SANTURIO, J. M. Micotoxinas e micotoxicoses na avicultura. Revista Brasileira de Ciência Avícola, Campinas, v. 2, p. 1-12, 2000.

SCAFF, R. M. C; SCUSSEL, V. M. Fumonisins B1 and B2 in Corn-Based Products Commercialized in the State of Santa Catarina - Southern Brazil. Brazilian Archives of Biology and Technology, Curitiba, v. 47, p. 911-919, 2004.

VAN DER WESTHUIZEN, L.; SHEPHARD, G. S.; SCUSSEL, V. M.; COSTA, L. L. F.; VISMER, H. F.; RHEEDER, J. P.; MARASAS, W. F. O. Fumonisin contamination and Fusarium incidence in corn from Santa Catarina, Brazil. Journal of Agricultural and Food Chemistry, Easton, v. 51, p. 5574-5578, 2003. 